\title{
Potential of intestinal microbiota profile as a diagnostic biomarker of small bowel transplant rejection
}

T

he need to identify a noninvasive marker that is predictive of intestinal allograft rejection may be a step closer to being met, thanks to the finding that small bowel transplant rejection is associated with changes in the intestinal microbiota.

"The transplanted gut is a fascinating and extreme situation, in which the host is trying to reject this large immunostimulatory organ and also confronting the microbes that live in the lumen of the gut," explains Daniel Peterson, corresponding author of the study. "The obvious question to ask was 'Are there specific changes in the ileal microbial community that are specific to rejection?"'

To try to answer this question, the team of researchers analyzed 35 ileal effluent samples taken from 19 small bowel transplant recipients (mostly pediatric) before surgical closure of their ileostomy. Symptoms, ostomy output, endoscopic and histologic findings and the absence of confounders (for example, viral enteritis) were used to classify the samples as rejecting or non-rejecting. For most of the patients who experienced rejection, ostomy samples taken within 2 weeks prior to rejection were available (a time when the ostomy output was normal and there were no symptoms suggestive of rejection)these samples were labeled as pre-rejecting.

To avoid potential bias being introduced by culturing the bacteria present in the ileal effluent samples, the researchers instead extracted bacterial DNA from the samples and used PCR to amplify the $16 \mathrm{~S}$ ribosomal RNA gene, which is highly conserved amongst different species of bacteria. The resulting so-called amplicon mixtures were then subjected to pyrosequencing-a next-generation sequencing method that allows a single strand of DNA to be sequenced. Next, these sequences (around 5,000 per sample) were analyzed using the Ribosomal Database Project Classifier tool and pyrosequencing pipeline (available via
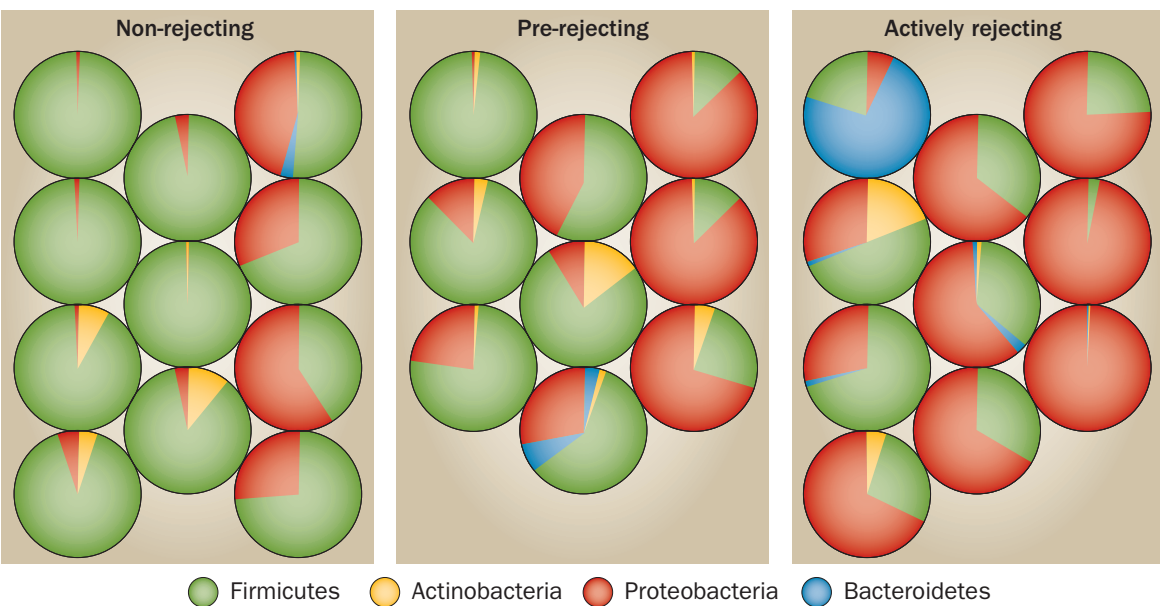

Bacteroidetes

Phylum level microbial composition of non-rejecting, pre-rejecting and actively rejecting ileostomy output samples taken from small bowel transplant recipients (minor taxa omitted for simplicity). Each pie chart represents the microbial composition of a single sample. Courtesy of D. Peterson.

http://rdp.cme.msu.edu/index.jsp) to see which types of bacteria dominated in the non-rejecting, pre-rejecting and actively rejecting ileal effluent samples.

At the phylum level, Firmicutes and Proteobacteria dominated the ileal microbiota of the small bowel transplant recipients; however, the proportion of these phyla differed according to rejection status. During the rejection process there was a significant reduction in Firmicutes (from $81 \%$ to $29 \%$ ), mostly attributable to a reduction in the order Lactobacillales, families Streptococcaceae, Enterococcaceae and Lactobacillaceae. There was also a significant increase in Proteobacteria (from 16\% to 61\%) that was mainly due to expansion of the order Enterobacteriales, family Enterobacteriaceae; the sum of the proportions of genera Escherichia and Klebsiella also increased significantly.

The team ruled out the influence of antibiotics or total bacterial count on their findings, and tested the potential of microbiota profiling as a diagnostic tool for detecting and predicting rejection. Their ROC analysis showed that the relative proportions of various bacterial taxa could discriminate between non-rejecting and actively rejecting samples. For Firmicutes, a cut-off value of $<49.7 \%$ identified active rejection with $90.0 \%$ sensitivity and $90.9 \%$ specificity.

So, what is next for Peterson and colleagues? "First, we need to determine if monitoring the microbiota can help identify the onset of rejection episodes and help the medical management of these patients in treating rejection or infections to improve the health of the allograft. Second, the implication of this work is that if we could suppress the growth of $E$. coli and/or Klebsiella types of bacteria and promote the Firmicute types of bacteria, then we might use probiotic, prebiotic or antibiotic approaches to promote a healthy microbiota and suppress pathologyassociated microbes in these patients."

Another question to be answered by future work is whether the changes in the microbiota are the cause or the result of the rejection process.

Natalie J. Wood 